\title{
SIMULATION OF THE IMPACT OF A DETERIORATED HIGH-PRESSURE COMPRESSOR ON THE PERFORMANCE OF A TURBOFAN ENGINE USING A PSEUDO BOND GRAPH MODELLING APPROACH
}

\author{
Jan Göing, Sebastian Lück, Christoph Bode, Jens Friedrichs \\ Institute of Jet Propulsion and Turbomachinery \\ Technische Universität Braunschweig \\ j.goeing@ifas.tu-braunschweig.de \\ Braunschweig, Germany
}

\begin{abstract}
The impact of a deteriorated high-pressure compressor in a bypass jet propulsion engine is investigated and simulated in this paper by using a pseudo bond graph approach. This theory is realised in the in-house software tool ASTOR (AircraftEngine Simulation of Transient Operation Research) to simulate the performance of a jet engine in a transient load case with dynamic and individual volumes. Furthermore, experimental test data of a pass off test run from idle and takeoff stationary operating points of the used in-house turbofan engine V2500-A1 are analysed and compared to the performance model's results. The engine blades are in a deteriorated state for the test run. In ASTOR, two cases are considered for modelling the V2500. They only differ in the use of either a new or a deteriorated high-pressure compressor to analyse their influence on overall performance. In general, the deviations between both ASTOR cases and experimental data are smaller with a deteriorated compare to the new HPC in the stationary operating points. However, some of the occurring effects can be explained with the components that remained unchanged within the model. In the transient load case, which is simulated in ASTOR, the performance of the deteriorated model shows an increase of the transient peaks of the exhaust gas temperature of up to $5 \%$.

In a nutshell, the HPC of a performance digital twin of a new V2500-A1 is replaced by for a deteriorated HPC of the in-house engine to investigate the influence on overall performance as well as to compare the results to the real jet engine in stationary operating points. With this information an earlier recognition of the deteriorated component is possible during maintenance, repair and overhaul (MRO) events.
\end{abstract}

\section{Introduction}

The maintenance and regeneration of a complex capital good, such as a jet engine, is an intensive and empirical process. However, to improve the diagnosis process and to understand the influence of the deteriorated components, it is highly relevant to model the interactions, impacts and performances of the isolated components and of the overall system. For more details, Li (2002) gives a review about performance analysis for gas turbines.

The Collaborative Research Centre (CRC) 871 "Regeneration of Complex Capital Goods" investigates the scientic bases for maintenance, repair and overhaul (MRO) to develop new approaches. One working group of the CRC 871 develops a virtual regeneration process to understand the impact of deteriorated and repaired components on the overall performance in more detail. For this purpose, the Institute of Jet Propulsion and Turbomachinery (IFAS) at Technische Universität Braunschweig develops a software tool called ASTOR (AircraftEngine Simulation for Transient Operation Research) to investigate the system performance in transient load cases.

The software tool ASTOR is based on the pseudo bond graph methodology. Benefits of this theory are a clear and general representation of the miscellaneous engine components with the ability to include geometrical information of individual dynamic volumes for the inertia and capacity of the jet engine (see Göing et al. (2019)). In contrast to constant mass flow methods, such as GasTurb, a transient manoeuvre can be calculated with higher accuracy by using the momentum equation and individual, dynamic volumes. The complete process can be described with a matrix of differential equations. Further expansions of an existing model are clearly defined by the bond graph notation, which is necessary to create a digital twin with all subsystems and side effects. This provides well-defined placement instructions for the connection of model/subsystem and the base model. A 
general development and more details of the bond graph theory are described in Karnopp et al. (2012).

In this scientific paper the performance of the turbofan engine V2500-A1 by International Aero Engines (IAE) is studied and modelled with the pseudo bond graph theory. Initially, the transformation of the jet engine into the performance model ASTOR is described. This model requires the geometry of each component (cross-sections, surface areas, lengths and weights) and divides the engine into different subsystems. Each subsystem is characterised by the pseudo bond graph theory that includes the equations of motion and inertia as well as capacity and friction of different mechanical and thermodynamic subsystems. Characteristic control volumes are required to define inertia and capacity. Furthermore, the pseudo bond graph notation of the engine's secondary air system and local heat flows is presented.

In addition, the implementation of thermodynamic gas tables in ASTOR to enhance accuraccy of the joule process is explained. In the next step, the steady state performance maps which are included in ASTOR, as well as an example of the performance map interpolation technique are presented.

The main focus of this publication is to find out the impact of an used high-pressure compressor (HPC) compared to an unused $\mathrm{HPC}$ on the overall performance in a steady state operating point as well as transient process.

In ASTOR, two different performance maps of the V2500A1 HPC are included. The first test case describes the new and unused HPC of the V2500-A1. The second condition characterises the compressor of the in-house V2500-A1 of the IFAS, which was investigated by Reitz et al. (2018).

Moreover, experimental data from a pass off test run of the in-house engine are used to compare the steady-state results of the two cases. Afterwards, selected results of the transient process are compared and validated with commercial software GasTurb by Kurzke (2012), which is being used by the CRC 871. Other performance tools are e.g. Numerical Propulsion System Simulation (NPSS) or Gas Turbine Laboratory (GTLab), which are not considered in this investigation. For the remainder of the study, only the acceleration manoeuvre from idle to takeoff is analysed with ASTOR. A detailed description of this manoeuvre can be found in Walsh and Fletcher (2004). Therefore, the temperature, pressure, thrust and spool speed are evaluated. These information are useful for a pre-prediction and early recognition of a deteriorated compressor.

\section{Methods}

In this section the properties, characteristics and techniques of the performance models are described in more detail. Furthermore, the slam acceleration of the real engine and the performance models are defined.

\section{Performance model of the V2500-A1}

In ASTOR, as well as in GasTurb, the V2500-A1 is divided into inlet, fan, booster (LPC), high-pressure compressor (HPC), combustion chamber (CC), high-pressure turbine (HPT), lowpressure turbine (LPT) and outlet of the core and bypass (see figure 1). While GasTurb uses a mixed nozzle, a separated nozzle is implemented in ASTOR. The included performance maps are the same and a burner time constant of 0 is applied in both tools.

In ASTOR, the pseudo bond graph theory is applied, which divides all quantities in efforts $e$ and flows $f$ to connect all different systems and transfer them into a system of differential equations. The equations of motion (see eqn. (1)-(3)) are solved in the 0 and 1 - junctions (see figure 1) to simulate transient performance. A detailed description about ASTOR can be found in Göing et al. (2019) and for a general consideration in Krikelis and Papadakis (1988).

In contrast to the established iterative and classic methods in performance simulation, only the following equations (1)-(3) are solved with the Bogacki Shampine 3th order solver and a sampling rate of $100 \mathrm{kHz}$. In the transient load case no beta - or $\mathrm{R}$ - lines are necessary. The calculated mass flow and rotating speed (see eqn. (3) and (7)) are used as input parameters for the compressor and turbine performance maps to obtain the pressure ratio $\pi$ and efficiency $\eta$.

$$
\begin{aligned}
\frac{\mathrm{d} p_{t o}}{\mathrm{~d} t}= & \frac{1}{C} \cdot\left(\dot{m}_{i}-\dot{m}_{o}-\dot{m}_{s a}\right) \\
\frac{\mathrm{d} T_{t o}}{\mathrm{~d} t}= & \frac{1}{C_{T}} \cdot\left(\dot{m}_{i} \cdot c_{p} \cdot\left(T_{t i}+\Delta T_{t}\right)-\dot{m}_{o} \cdot c_{p} \cdot T_{t o}\right. \\
& \left.-\dot{m}_{s a} \cdot c_{p} \cdot T_{t o}-Q_{i}\right) \\
\frac{\mathrm{d} \dot{m}}{\mathrm{~d} t}= & \frac{A D}{L} \cdot\left(p_{i}+\Delta p-p_{R}-p_{o}\right)=\frac{1}{I} \cdot\left(p_{i}+\Delta p-p_{R}-p_{o}\right)
\end{aligned}
$$

Ducts are implemented in the bond graph diagram to connect the secondary air system, such as cooling air, bleeds or leaks, to the main gas path. As an example, the cooling air system is shown in the expansion of figure 1 between HPC, HPT and LPT. In a transient load case the massflow within the main gas path and the cooling flow can be calculated through the equations of motion. Due to the unknown pressure and geometrical boundary conditions a constant relative cooling air flow $\dot{m}_{s a}$ between compressor and turbine is defined for each operating point, which is calculated with a constant resistor $R_{s a}=0.11$ (see eqn. (4) \& (5)). In addition, local heat flows between the bypass and core as well as between the material and the gas flow are highly relevant. In this publication, these local heat flows currently remain neglected. However, in the bond graph diagram (see figure 1) an example of the heat flows is shown between the environment, 
bypass and core flow. The temperature and heat flows can be calculated with eqn. (5) and (6). The resistor $R$ for the heat flow depends on the area A between fluid and material and the averaged heat transfer coefficient $\bar{\alpha}$, which is a function of the NusseltNumber (see Dittus and Boelter (1985) and Schobeiri (2017)). An example of the local heat flows in stationary gas turbines modelled with the pseudo bond graph theory can be found in Krikelis and Papadakis (1988).

$$
\begin{aligned}
\dot{m}_{s a} & =\frac{\dot{m}_{o}}{R_{s a}} \\
Q & =\frac{1}{R_{w}}\left(T_{t}-T_{W t}\right)=\bar{\alpha} \cdot A \cdot\left(T_{t}-T_{W t}\right) \\
\frac{\mathrm{d} T_{W t}}{\mathrm{~d} t} & =\frac{1}{C_{T W}} \cdot\left(Q_{i}-Q_{o}\right)=\frac{1}{m_{W} \cdot c_{p W}}\left(Q_{i}-Q_{o}\right) .
\end{aligned}
$$

The spool speed $\omega$ is determined using the difference between turbine and compressor torque $\tau$. Furthermore, the inertia of the spool is necessary (see eqn. (7)). The geometries of both spools are simplified to measure and calculate the moments of inertia $J$. Both systems are estimated with cones, conical frustum and hollow cylinders. The volumes are then combined with the density of the materials used which can be found in the literature. The rotor blades are simplified as symmetric point masses. The resulting moment of inertia for $\omega_{L P}$ is approximately $85 \mathrm{~kg} \cdot \mathrm{m}^{2}$ - and of the $\omega_{H P}$-spool is around $15 \mathrm{~kg} \cdot \mathrm{m}^{2}$.

$$
\frac{\mathrm{d} \omega}{\mathrm{d} t}=\frac{1}{J} \cdot\left(\tau_{T}-\tau_{C}-\tau_{R}\right)
$$

In ASTOR and GasTurb the same performance maps for all turbo machines are applied. The performance maps of the new and deteriorated HPC can be found in Reitz et al. (2018), which are used to investigate the two different cases. The first case investigates the performance with a new HPC map and the second represents a deteriorated HPC.

In the following subsection the interpolation technique of the performance maps is depicted using the HPC. Furthermore, general scaled performance maps are applied for the Fan, LPC, HPT and LPT (see Kurzke (2012)).

\section{Performance maps}

Performance maps feature a limited set of measured or simulated data points at few rotational speeds. ASTOR uses performance maps to obtain pressure ratios and efficiencies at given massflows and speeds to calculate $\Delta p$ in equation (3). Points which are not directly on given characteristic curves have to be interpolated. To guarantee a stable algorithm even in operating areas of the performance map with pronounced gradients, e.g. at the right of the turbine map, where flow chokes the resolution of points along the characteristic curves, has to be augmented. The same applies to the number of characteristic curves which are the starting point of interpolation. To increase the resolution in both dimensions mentioned, an algorithm is

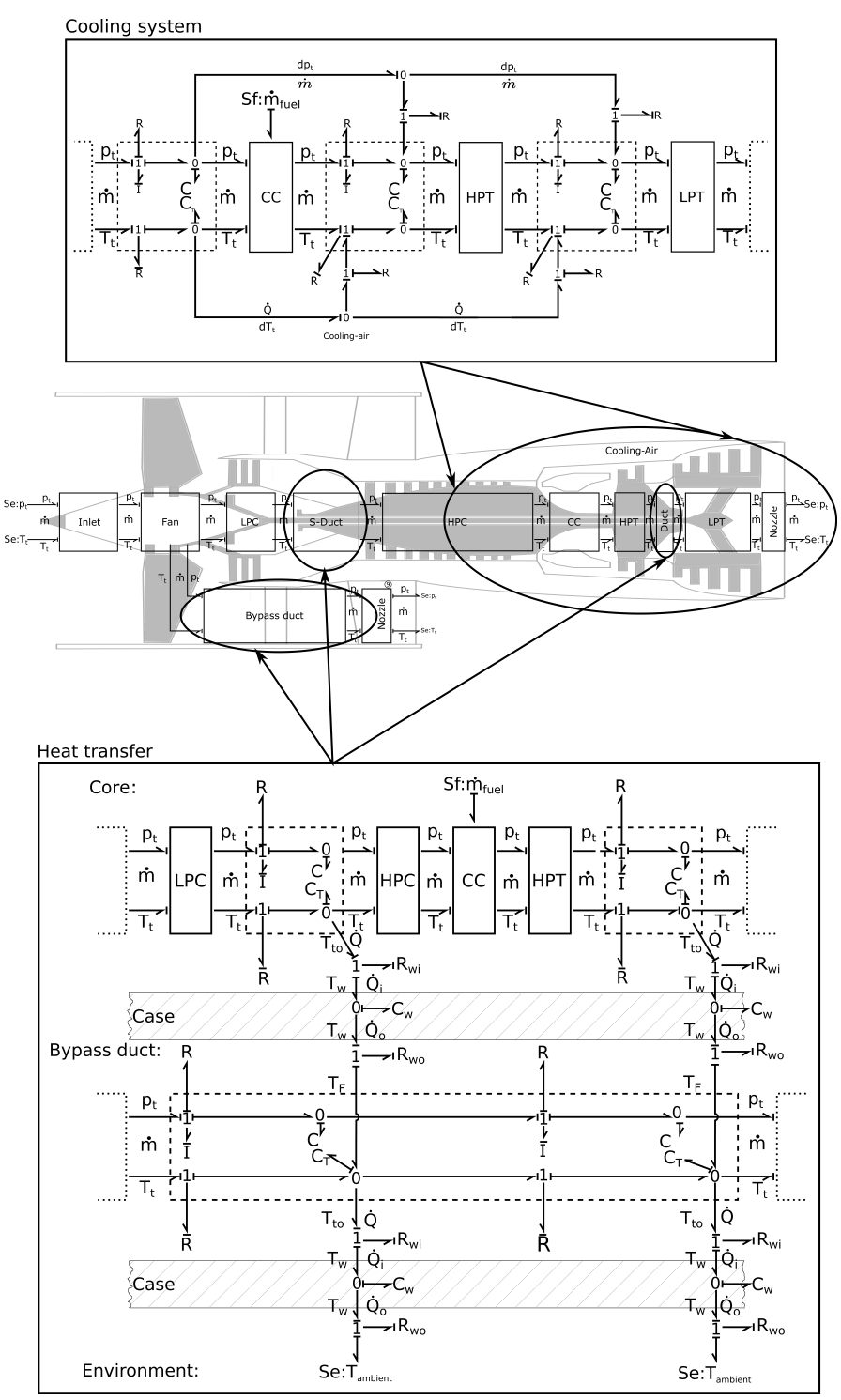

FIGURE 1. V2500 - A1 turbofan jet engine; centre stage: pseudo bond graph with duct, friciton, secundary air system and local heat flow (transfer); background: sketch of the V2500-A1.

proposed to initially bring performance maps into a uniform shape with an equal number of points along each characteristic curve. This is accomplished by fitting a piecewise hermite spline (Fritsch and Carlson (1980)) through the given datapoints to obtain a polynomial description of the characteristic curves which can then be evaluated on a given massflow range at the desired resolution, thus increasing the resolution of data points. The second dimension requires the interpolation of additional characteristic curves into the space between two given ones for a desired rotational speed. The first assumption can therefore be a linear interpolation between the previously generated i-th point on the neighbouring lines which uses the rotational speed as the linear weighing factor. In accordance to Gharaibeh and Costall (2017) a characteristic curve of the given dataset is deleted and then re-interpolated by applying the described algorithm, to validate the proposed scheme and to obtain the accuracy estimates. The results of the linear approach are shown in figure 2 in red 


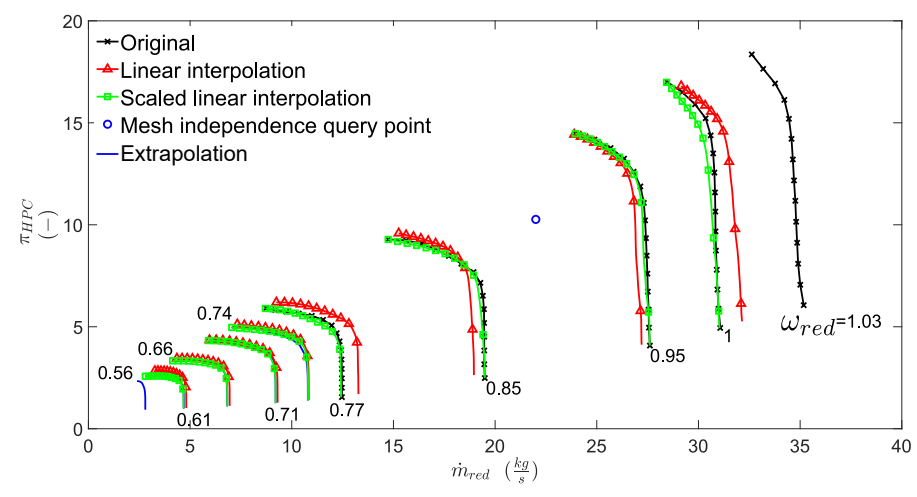

FIGURE 2. Validation of performance map interpolation algorithms - Comparison of linear and linear scaled interpolation for the reinterpolation of a deleted characteristic curve within the performance map.

as re-interpolated characteristic curves wihthin the original performance map. While the relations show the overall plausibility in comparison to original data, it can be observed that a linear relationship of $\pi, \dot{m}$ and $\omega$ is not an adequate assumption along dedicated areas of the operating range. Therefore, to improve the technique the performance map's limits are used as functions of the type $\dot{m}=f(\omega)$ and $\pi=f(\omega)$ extracted from the map's choke and surge line, which are taken as the first and respectively the last value of each characteristic curve. While these do not match the actual limits of the operating range, usually no test data is available for the real limits as such experiments can potentially damage the machine. Using this approach for a given $\omega$, more adequate values can be obtained. These values are then used to scale the result of the linear interpolated speedline to the limits of the performance map. The results (green) show a better fit than those of a solely linear approach mentioned before due to taking into account nonlinear phenomena represented by the maps limits. A significant increase in accuracy can be achieved when employing the scaling approach. To conclude the required resolution of the performance map necessary to obtain a mesh independent solution a mesh independence study is conducted for each intra-characteristics interval (figure 3). Exemplary results are shown for a query point at $\dot{m}_{\text {red }}=22 \mathrm{~kg} / \mathrm{s}$ and $\omega_{\text {red }}=0.94$ which is shown in figure 2 as well. From these observations a mesh independent solution can be obtained if the resolution along the massflow axis is greater than 500 points and the number of interpolated speed lines in each interval is greater than 50. Equal or even lower values are obtained for the remaining intervals. The results of ASTOR in the following chapters are obtained using this resolution to ensure a mesh independent interpolation result. To simulate the test run from idle to takeoff all maps are enlarged by the extrapolation of characteristic curves for lower rotational speeds (see Kurzke (1996)). The extrapolated speed lines from 0.56 up to 0.77 are displayed in the performance map in figure 2 .

\section{Gas tables}

From a thermodynamic point of view the assumption of a calorically perfect gas is a major simplification. Due to real

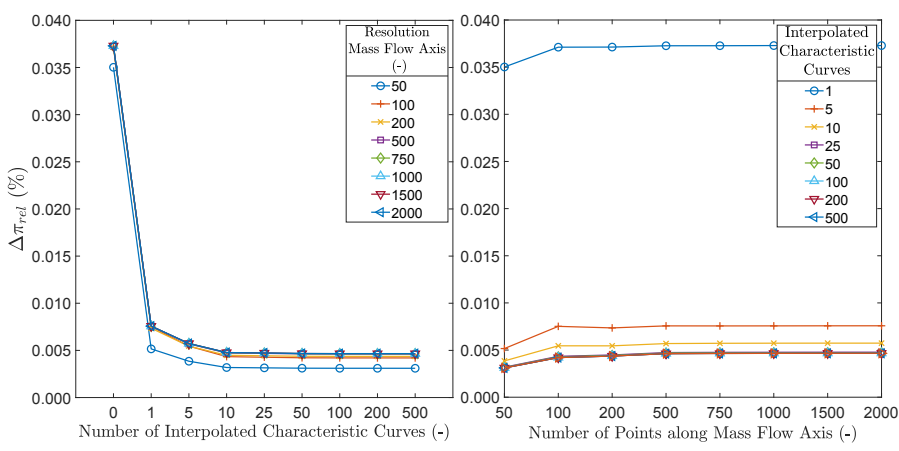

FIGURE 3. Grid convergence study for required performance map resolution. Relative deviation of the result in comparison to the coarsest resolution of massflow axis and number of interpolated characteristic curves respectively.

gas effects especially at high temperatures and pressure ratios, this assumption leads to discrepancies compared to testbed data. Therefore, real gas data has to be considered within performance simulations in ASTOR. The used data are based on polynomial approaches of Gordon and McBride (1994); McBride and Gordon (1996); McBride et al. (1993) which contain data of specific enthalpy $h$, heat capacity $c_{p}$ and an entropy function $\Gamma$ for a given temperature $T$. The latter must not be confused for absolute entropy. To take real gas data into account, gas tables are calculated for a temperature range covering the entire flight envelope and temperatures occurring within the engine. Subsequently, during program execution for a component e.g. a compressor a table lookup is performed to gather appropriate data from the gas tables by calculating the entropy rise as a function of the compressor pressure ratio $\pi$ from the performance map which is used to calculate the isentropic enthalpy rise. Incorporating the isentropic efficiency from the performance map the real enthalpy rise can be calculated and thereby the specific work needed to perform the compression. From the final level of enthalpy the gas tables provide corresponding values of specific heat capacity and gas temperature which thereafter are used in the 0 - junction, eqn. (2) respectively, to obtain the $\Delta T$. In addition, the calculated specific heat capacity $c_{p}$ is used within this equation in the last term to account for a change of $c_{p}$ due to the change of state. This is especially necessary for large pressure rises such as those in a modern engines HPC of $\pi \approx 15$.

\section{Pass off test run of the V2500-A1 engine}

So far, a pass off test run of the in-house jet engine has been conducted to find out the current performance at stationary operating points, such as idle and takeoff. The V2500 jet engine has accumulated 18786 cycles since new. Further explanations of this engine can be found in Spuhler et al. (2019) and of this HPC in Reitz et al. (2018). In this study, a fuel flow signal of an acceleration between idle and takeoff is taken from test data, which is idealised and used as input for ASTOR.

In figure 4 the normalised fuel flow $\dot{m}_{f}^{*}$ over the normalised time $t^{*}$ is displayed. The measured fuel flow is shown by the dashed blue line while the input signal for the performance model is represented by the solid red line. The measured fuel flow can be divided in 4 notable areas. Until the first black line, in area A, an 


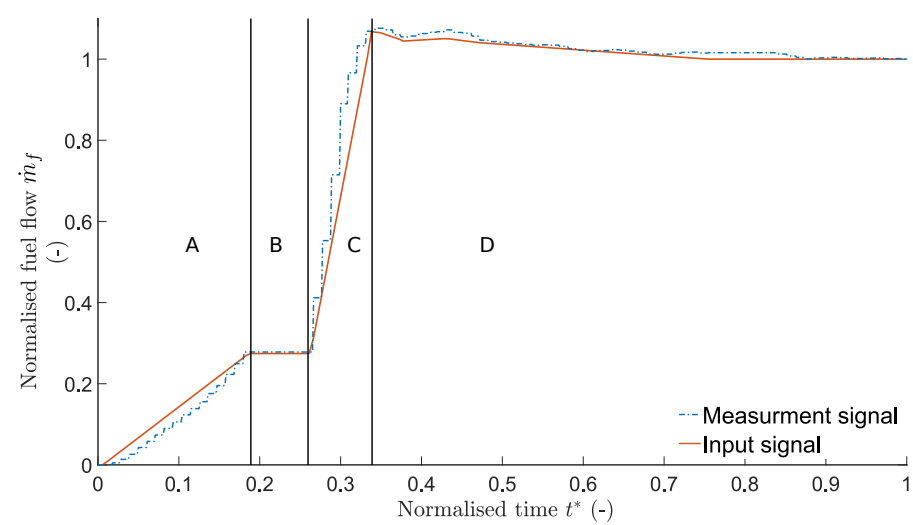

FIGURE 4. Normalised fuel flow $\dot{m}_{f}^{*}$ over the normalised time $\mathrm{t}^{*}$; Signal form the test data in dashed blue, input signal for the performance models in solid red line.

increasing fuel flow is visible. Afterwards, a constant fuel flow is detected in area B. In area C, the fuel flow rises up to the max. value and in area $\mathrm{D}$ it decrease until the final value. This signal was measured at two different measurement ports to obtain reliable data.

\section{Results and Discussion}

In this section, selected results of the test bed run are compared with the results of ASTOR. In ASTOR two cases are considered. In the first case the new HPC performance map is included and in the second case the map of the deteriorated HPC is considered. At the beginning, both cases are validated and compared to GasTurb. In the next step, the stationary operation points idle and takeoff of the test data are compared to the new and deteriorated HPC ASTOR model respectively. Finally, selected results of the transient load case are juxtaposed and discussed.

\section{Comparison between GasTurb and ASTOR}

A comparison to the GasTurb model is conducted to validate the unsteady ASTOR results qualitatively and to explain the advantages of the pseudo bond graph theory with dynamic volumes.

In figure 5 the normalised turbine inlet temperature $T I T^{*}$ over the normalised time $t^{*}$ is shown for the slam acceleration from idle to takeoff. The $T I T^{*}$ of the second ASTOR case (deteriorated HPC) is represented with solid red line with marker and the first ASTOR case (new HPC) with the solid blue line with pluses. On the other hand, the second case in GasTurb (deteriorated HPC) is shown with a dashed purple line and the first case in GasTurb (new HPC) with a yellow solid line. The general shape of the curves is similar for ASTOR and GasTurb. However, until the end of zone B a significant offset in GasTurb compared to ASTOR is visible. Furthermore, the transient peaks in GasTurb are instantaneous and occur at the same time as the change of the fuel flow (see figure 4), due to the constant mass flow in the single components. In ASTOR, a radius and a later reach of these peaks is visible due to the dynamic volumes. A deviation of the transient peak after the acceleration in area D of $2.5 \%$ is calculated in ASTOR and of $1.5 \%$ in GasTurb (see expansion in

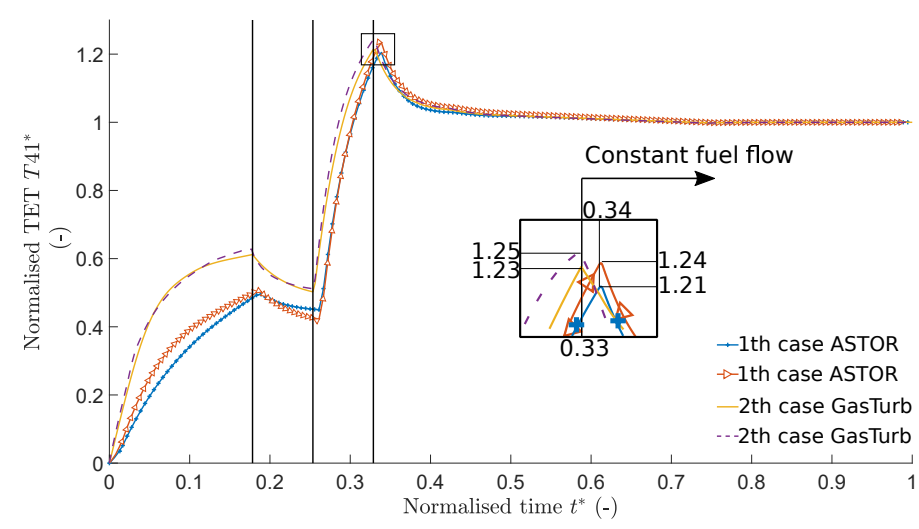

FIGURE 5. Normalised $T I T^{*}$ over the normalised time $t^{*}$; Comparisons between a new and deteriorated HPC in ASTOR and GasTurb; first case ASTOR (new HPC) in solid blue line with plus, second case ASTOR (deteriorated HPC) in solid red line with marker; GasTurb first case with solid yellow line, GasTurb second case with dashed purple line.

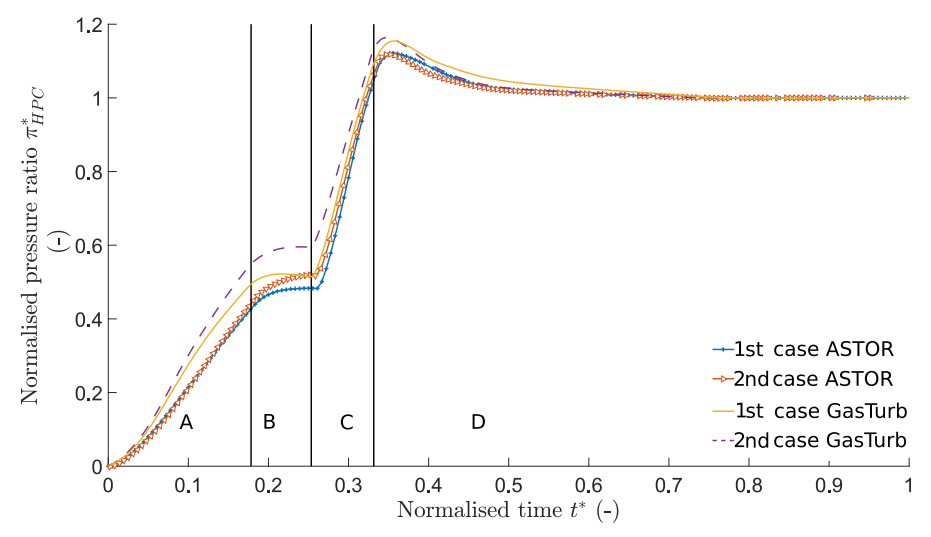

FIGURE 6. Normalised $\pi_{H P C}^{*}$ over the normalised time $t^{*}$; Comparisons between a new and deteriorated HPC in ASTOR and GasTurb; first case ASTOR (new HPC) in solid blue line with plus, second case ASTOR (deteriorated HPC) in solid red line with marker; GasTurb first case with solid yellow line, GasTurb second case with dashed purple line.

figure 5). Consequently, a significantly higher transient peak of the $T I T^{*}$ in both models can be detected.

In figure 6 the normalised pressure ratio $\pi_{H P C}$ over $t^{*}$ is plotted. Similar to the $T I T^{*}$, the step of the fuel flow can be recognised in $\pi_{H P C}$. In contrast to the temperature, the transient peaks of the pressure ratio are smoother and with lower amplitudes. In both performance tools the 2nd case shows a significant offset of the pressure ratio compared to the new one. Furthermore, the 2 nd case reaches the final pressure faster in both tools. The pressure rise of the 2 nd case shows a larger gradient without a significantly larger amplitude of the overshoot.

In both performance tools the general behaviour of the curves is similar. Only a significant offset is detected. Therefore, the validity of the in-house model can be concluded and only the results of ASTOR will be used in the next sections. 


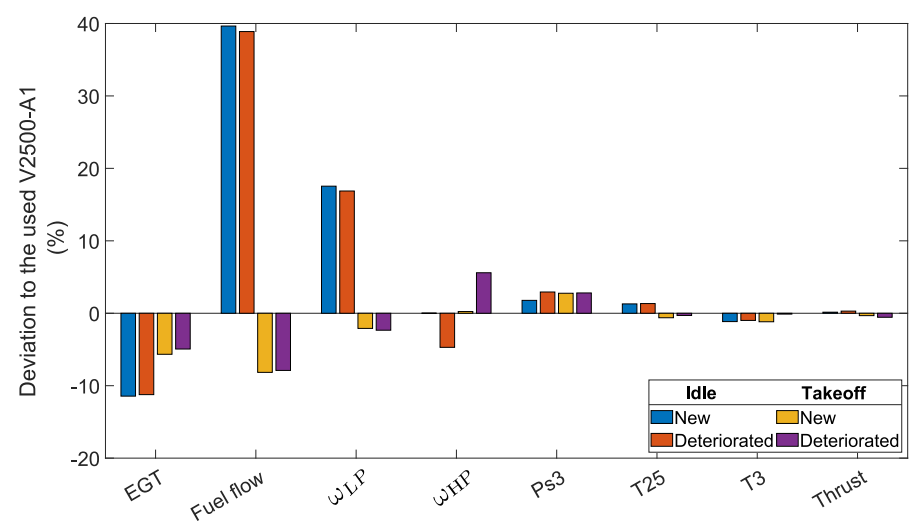

FIGURE 7. Deviation between the new and deteriorated HPC performance models of the V2500-A1 in idle and takeoff compared to the real test data of the used in-house jet engine.

\section{Investigation of the deteriorated HPC}

In figure 7 the deviations between the two cases and the experimental test data of the used V2500-A1 test engine in the steady state operating points idle and takeoff are displayed. The new HPC is shown with blue (idle) and yellow (takeoff) bars and the deteriorated HPC with the red (idle) and purple (takeoff) bars. The deviations of exhaust gas temperature $(E G T)$, fuel flow, $\omega_{L P}$, $\omega_{H P}, P_{s 3}, T_{25}, T_{3}$ and thrust are listed in this diagram. In general, deviations are in the range of $-12 \%$ up to $40 \%$ in idle and $-9 \%$ to $6 \%$ in takeoff. The largest differences are detected in the fuel flow (idle up to $40 \%$, takeoff up to $-8 \%$ ) and in $\omega_{L P}$ (idle up to $18 \%$ ). The thrust is the target value of the simulation to set up the models at equivalent operating points in comparison to the test run and therefore only differ negligibly. At idle the engine has a thrust of around $10 \mathrm{kN}$ and at takeoff $100 \mathrm{kN}$.

Most values show smaller deviations in the deteriorated case compared to the new HPC. However, the spool speed $\omega_{H P}$ has a significantly higher deviation in both operating points. This effect can be explained with the performance map of the deteriorated HPC while the maps of the remaining components remain unchanged. However, due to the large deviation in spool speed, the operating lines within the performance maps are different, which has an impact on all quantities along the stationary and transient working line. As mentioned in the chapter "Performance maps" the characteristic curves for idle conditions have been extrapolated to contain the idle operating point. Furthermore, the influence of variable stator vanes in this area is unknown which has a significant impact on the performance and on the fuel flow input.

However, the diagram shows a worsening of $0.5 \%$ in idle and 2 $\%$ in takeoff of the EGT. The static pressure $P_{S 3}$ after the HPC shows a lower deviation between new and real engine.

To complete the comparison between GasTurb and ASTOR the standard deviations in the four cases are in the range of $2-5 \%$.

\section{Transient manoeuvre}

In figure 8, 9, 10 and 11 cases 1 and 2 are compared to each other to identify the impact of the deteriorated HPC on the overall performance. The 1st case (new HPC) is presented with a solid blue line with pluses and the 2nd case (deteriorated HPC)

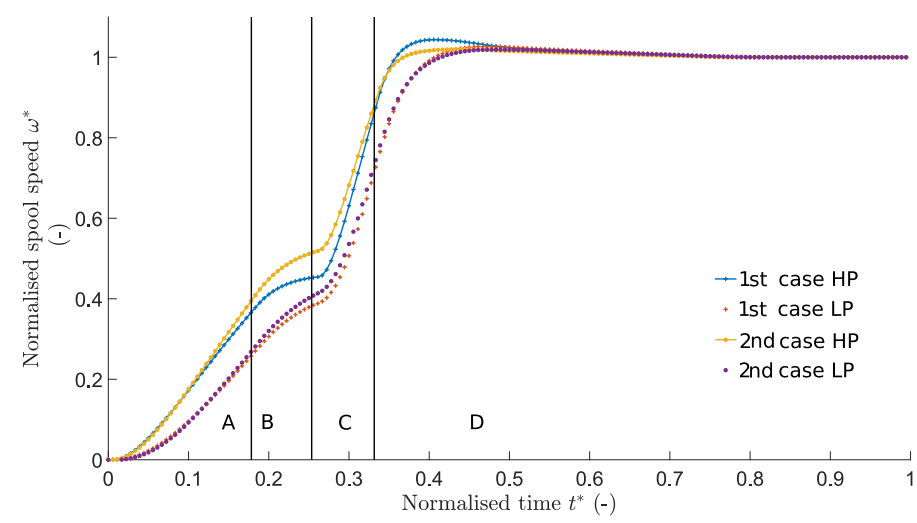

FIGURE 8. Normalised spool speed $\omega^{*}$ over the normalised time $t^{*}$; The data of ASTOR with the new HPC (1st case) are marked with pluses $\left(\omega_{H P}\right.$ with solid line); The data of ASTOR with the deteriorated HPC (2nd case) are marked with dots ( $\omega_{H P}$ with solid line).

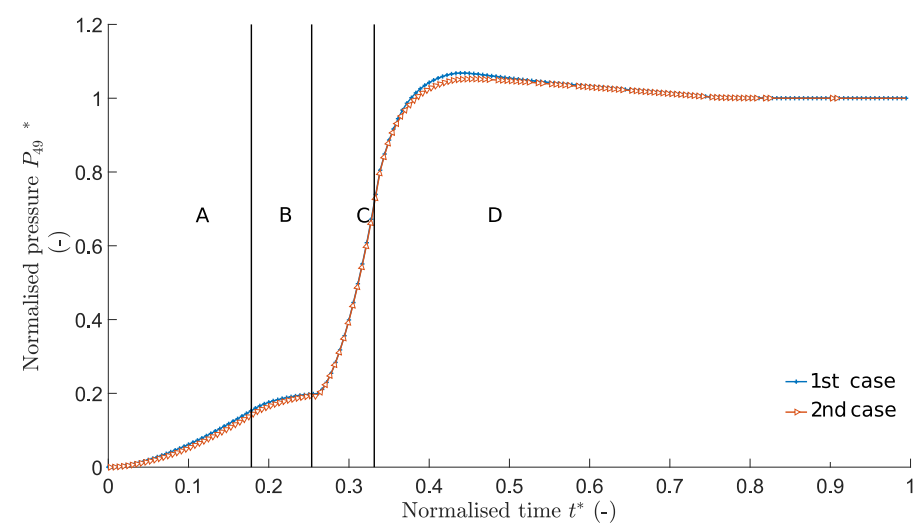

FIGURE 9. Normalised $P_{49}^{*}$ over the normalised time $t^{*}$; Comparisons between a new and deteriorated HPC in ASTOR; first case in solid blue line with pluses, second case in solid red line with marker.

with a solid red line with marker. The different areas, which are explained in figure 4 are equally highlighted.

The increasing speed of the high- and low-pressure spool is shown in figure 8 . In general, a strictly monotonous increase of both spool speeds is detected in all curves. The step of the fuel flow is visible in area B. Furthermore, at the end of the slam acceleration an overestimation in HPC spool speed occurs before it swings back to the final value. This peak is located at $t^{*}=0.4$. An offset of case 2 is identified up to the end of zone $C$.

In figure 9 the normalised pressure $P_{49}^{*}$ is plotted over $t^{*}$. The final $P_{49}^{*}$ of both simulations is reached at $t^{*}=0.75$. Furthermore, the fuel flow step is visible in all simulations. In the full manoeuvre, the $P_{49}^{*}$ of the second case is lower than $P_{49}^{*}$ of the first case. The max. deviation is $-0.5 \%$ at the last peak. In contrast to the $T I T$, the transient peak after the area $\mathrm{C}$ has a smaller amplitude in case 2.

In figure 10 the normalised thrust $F N^{*}$ is shown over $t^{*}$. The thrusts increase after $t^{*}=0.01$ until 0.4. After that time, the thrusts decreases slowly to the final value.

The step of the fuel flow in area B can be identified in the results. Moreover, no significant deviation between the different cases can be recognised. Consequently, the impact of the deteriorated 


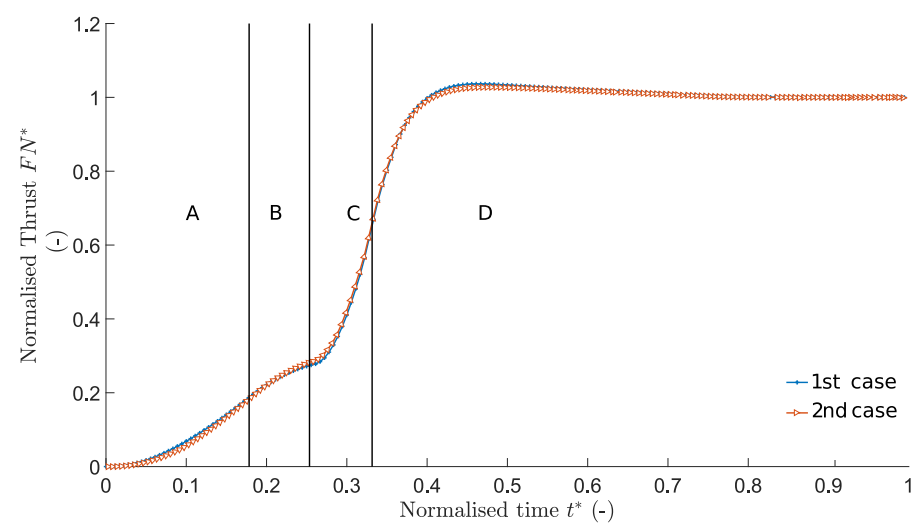

FIGURE 10. Normalised $F N^{*}$ over the normalised time $t^{*}$; Comparisons between a new and deteriorated HPC in ASTOR; first case in solid blue line with pluses, second case in solid red line with marker.

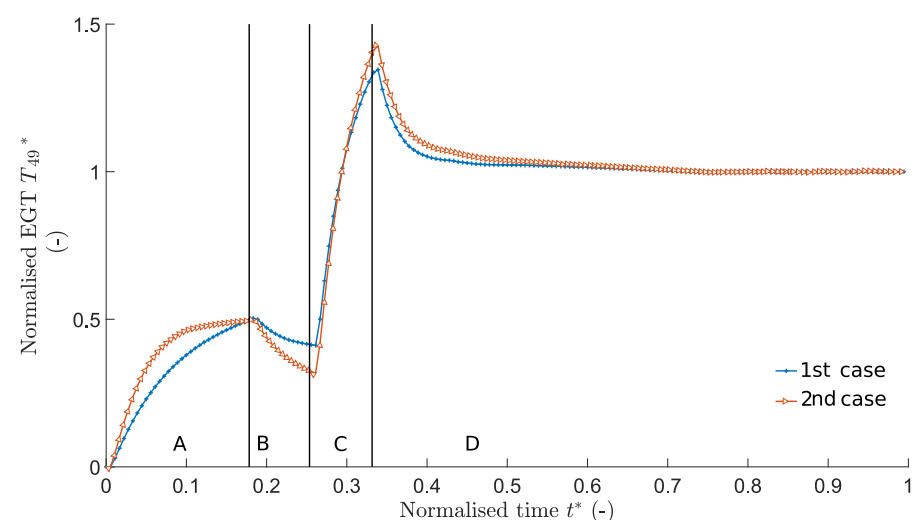

FIGURE 11. Normalised $E G T^{*}$ over the normalised time $t^{*}$; Comparisons between a new and deteriorated HPC in ASTOR; first case in solid blue line with pluses, second case in solid red line with marker.

HPC on the thrust during a transient load case can be neglected.

In figure 11 the normalised $E G T^{*}$ is shown over $t^{*}$. The deviation of the EGT at the stationary operating points can be found in figure 7. In general, a similar behaviour of the curves of the simulations is visible in figure 11. The step of the fuel flow is visible and the EGT and TIT of the 2nd case show higher amplitudes in the transient peaks. The deviation between models is about $5 \%$ in the last peak. Furthermore, a higher gradient in all areas of the deteriorated model is detected. At constant fuel flow in area B the $E G T^{*}$ decreases by $25 \%$ more in case 2 compared to case 1 , which is a significant deviation in the transient peaks.

\section{Conclusions and Outlook}

In this study the impact of a deteriorated HPC on the overall performance of a jet engine is investigated and compared to test bed data of an used engine. The performance at steady state operation is studied at the stationary operating points idle and takeoff. Furthermore, the acceleration between these points is investigated to show the impact of the deteriorated compressor. For this investigation the in-house tool ASTOR is used, which is based on the pseudo bond graph theory. This theory enables the consideration of the momentum equation with dynamic and individual volumes, which allow for a higher degree of detail when conducting transient simulations. The simulations are taking cooling air into account.

At stationary operating points a small deviation between the used engine and the performance model with the deteriorated HPC is detected compared to the new engine. Especially the deviations of EGT, TIT and $T_{3}$ are smaller. Large deviations in the fuel flow and spool speed are explainable due to the simplifications and assumptions necessary at the idle operating point in the simulations.

Furthermore, the spool speeds, important temperatures, pressures and the total thrust are investigated in a slam acceleration between idle and takeoff. The amplitudes of transient peaks in the temperature are significantly higher in the case with a deteriorated HPC. Deviations of up to $5 \%$ within the transient peaks are detected.

On the other hand, an amplitude of the deteriorated model of about $-1 \%$ is identified in the transient peaks of the pressure curves. Finally, no significant deviations in the thrust levels are identified. Therefore, a deteriorated HPC increases the temperatures, decreases the pressures and has no significant impact on the thrust in the stationary operating points and the transient load case under investigation.

In general, the deviation between deteriorated model and real used jet engine are small. However, some major differences are visible between the models and the test run data. Certainly, the most relevant reason is the use of general scaled performance maps of the remaining turbo machine components and the extrapolation to include the idle point. Due to the unknown type of deterioration, the results of the study refer only to the HPC of the IFAS V2500-A1 jet engine. In future studies, local heat flows will be considered in more detail. These flows have a significant impact on the performance of the TIT and EGT in an acceleration between idle and takeoff. Furthermore, different specific signs of wear and effects of deterioration of the HPC will be investigated to understand the impact of friction, material abrasion inside of the HPC on the overall performance. With the resulting knowledge a more general statement regarding the performance impact will be possible.

Moreover, the CRC 871 investigates the deterioration and geometry variances of the HPT as well as the impact on the overall system in a transient manoeuvre.

Finally, the vanes and rotors of the HPC will be digitised to find out more information of the deterioration in detail. A validation test run is planned to compare ASTOR in different manoeuvres to new test data of stationary operating points as well as transient manouvres. 


\section{NOMENCLATURE}

$A D$ Actuator disk

$\bar{\alpha}$ Averaged heat transfer coefficient

$C$ Capacity element

$C_{T}$ Thermodynamic accumulator

$C_{T W}$ Capacity element for heat flow

$c_{p} \quad$ Specific heat coefficient

$\Delta T \quad$ Temperature rise

$\Delta p \quad$ Pressure rise

$e$ Effort

$f$ Flow

$\Gamma$ Entropy function

FN Thrust

$\mathrm{h}$ Specific enthalpy

I Inertia element

$i, o$ In-/ outcoming

$J$ Inertia

$L \quad$ Characteristic length

m Mass

$\dot{m}$ Massflow

$P$ Pressure

$P_{s 3} \quad$ Static pressure after the HPC

$\pi$ Pressure ratio

$Q$ Heat flow

red Reduced value

$R$ Specific gas constant

$R, R_{s a} \quad$ Resistor

$\rho$ Density

sa Secondary air system

$s$ Specific entropy

$T$ Temperature

$T_{25}, T_{3}$ Temperature in front of and after the HPC

$\tau$ Torque

$V$ Volume

$W \quad$ Wall

$\omega$ Rotation speed

ASTOR AircraftEngine Simulation of Transient Operation Research

CC Combustion Chamber

CRC Collaborative Research Centre

IFAS Institute of Jet Propulsion and Turbomachinery

HPC High-pressure compressor

HPT High-pressure turbine

LPC Low-pressure compressor

LPT Low-pressure turbine

MRO Maintenance, repair and overhaul

TEC Turbine exhaust case

$\pi^{*}=\frac{\pi-\pi_{\text {start }}}{\pi_{\text {end }}-\pi_{\text {start }}} \quad$ Normalised pressure ratio

$\dot{m}^{*}=\frac{\dot{m}-\dot{m}_{\text {start }}}{\dot{m}_{\text {end }}-\dot{m}_{\text {start }}} \quad$ Normalised massflow

$\dot{m}_{f}^{*}=\frac{\dot{m}_{f}-\dot{m}_{f s t a r t}}{\dot{m}_{\text {end }}-\dot{m}_{\text {fstart }}} \quad$ Normalised fuel flow

$P_{49}^{*}=\frac{P_{49}-P_{49 \text { start }}}{P_{49 \text { end }}-P_{49 \text { start }}} \quad$ Normalised pressure after the LPT

$t^{*}=\frac{t-t_{\text {start }}}{t_{\text {end }}-t_{\text {stat }}} \quad$ Normalised time

$T I T^{*}=\frac{T I T-T I T_{\text {start }}}{\text { TIT }_{\text {end }}-\text { TIT }_{\text {start }}} \quad$ Normalised turbine inlet temperature

$T_{49}^{*}, E G T^{*}=\frac{E G T-E G T_{\text {start }}}{E G T_{\text {end }}-E G T_{\text {start }}} \quad$ Normalised exhaust gas temperature

$\omega_{h p}^{*}=\frac{\omega_{h p}-\omega_{\text {hpstart }}}{\omega_{\text {hpmax }}-\omega_{\text {hpstart }}} \quad$ Normalised rotation speed of the hp spool $\omega_{l p}^{*}=\frac{\omega_{l p}-\omega_{l p s t a r t}}{\omega_{l p m i n}-\omega_{l p s t a r t}} \quad$ Normalised rotation speed of the lp spool

\section{ACKNOWLEDGMENTS}

The authors kindly thank the German Research Foundation (DFG) for the financial support to undergo the research project D6 "Interaction of combined module variances and influence on the overall system behavior" within the Collaborative Research Center (CRC) 871 - Regeneration of Complex Capital Goods.

\section{ORCID ID}

Jan Göing (D) orcid.org/0000-0002-1279-1933

Sebastian Lück (D) orcid.org/0000-0003-1696-1300

\section{References}

Dittus, F. and Boelter, L. (1985). Heat transfer in automobile radiators of the tubular type, International Communications in Heat and Mass Transfer 12(1): 3-22.

Fritsch, F. N. and Carlson, R. E. (1980). Monotone piecewise cubic interpolation, SIAM Journal on Numerical Analysis 17(2): 238-246.

Gharaibeh, K. and Costall, A. W. (2017). A flow and loading coefficientbased compressor map interpolation technique for improved accuracy of turbocharged engine simulations, SAE Technical Paper Series, SAE International.

Göing, J., Kellersmann, A., Bode, C. and Friedrichs, J. (2019). Jet Propulsion Engine Modelling using Pseudo Bond Graph Approach, number GT2019-90420, Proc. ASME Turbo Expo, Paper No. GT2019-90420.

Gordon, S. and McBride, B. J. (1994). Computer program for calculation of complex chemical equilibrium compositions and applications. part 1: Analysis.

Karnopp, D. C., Margolis, D. L. and Rosenberg, R. C. (2012). System dynamics: modeling, simulation, and control of mechatronic systems, John Wiley \& Sons.

Krikelis, N. and Papadakis, F. (1988). Gas turbine modelling using pseudo-bond graphs, International journal of systems science 19(4): 537-550.

Kurzke, J. (1996). How to get component maps for aircraft gas turbine performance calculations, ASME 1996 International Gas Turbine and Aeroengine Congress and Exhibition, American Society of Mechanical Engineers, pp. V005T16A001-V005T16A001.

Kurzke, J. (2012). Gasturb 12: A program to calculate design and off-design performance of gas turbines. users manual.

Li, Y. G. (2002). Performance-analysis-based gas turbine diagnostics: A review, Proceedings of the Institution of Mechanical Engineers, Part A: Journal of Power and Energy 216(5): 363-377.

McBride, B. J. and Gordon, S. (1996). Computer program for calculation of complex chemical equilibrium compositions and applications ii. users manual and program description. 2; users manual and program description.

McBride, B. J., Gordon, S. and Reno, M. A. (1993). Coefficients for calculating thermodynamic and transport properties of individual species.

Reitz, G., Dwinger, K., Schlange, S., Friedrichs, J. and Kappei, F. (2016). Analysis of jet engine compressor deterioration and capturing correlations between geometric parameters, 16th International Symposium on Transport Phenomena and Dynamics of Rotating Machinery.

Reitz, G., Kellersmann, A. and Friedrichs, J. (2018). Full high pressure compressor investigations to determine aerodynamic changes due to deterioration, ASME Turbo Expo 2018: Turbomachinery Technical Conference and Exposition, American Society of Mechanical Engineers, pp. V02AT39A035V02AT39A035.

Schobeiri, M. T. (2017). Gas Turbine Design, Components and System Design Integration, Springer.

Spuhler, T., Kellersmann, A., Bode, C.and Friedrichs, J., Reitz, G., Kotulla, M., Kappei, F. and Michaelis, T. (2019). The v2500-a1 as a test rig towards digital twin modeling, International Gas Turbine Congress 2019 Tokyo, 17.-22. November 2019 (accepted).

Walsh, P. P. and Fletcher, P. (2004). Gas turbine performance, John Wiley \& Sons. 